\title{
Characterization of the radiation tolerance of cryogenic diodes for the High Luminosity LHC inner triplet circuit
}

\author{
Andreas Will" \\ CERN, Geneva 1211, Switzerland \\ and Karlsruhe Institute of Technology (KIT), Karlsruhe 76131, Germany
}

(within the High Luminosity LHC Project)

\begin{abstract}
G. D’Angelo, R. Denz®, D. Hagedorn, A. Monteuuis®, E. Ravaioli, F. Rodriguez Mateos, A. Siemko, K. Stachon, A. Verweij॰, and D. Wollmann $\odot$

CERN, Geneva, Switzerland
\end{abstract}

A.-S. Mueller and A. Bernhard

Karlsruhe Institute of Technology (KIT), Karlsruhe 76131, Germany

(Received 7 February 2020; accepted 11 May 2020; published 27 May 2020)

\begin{abstract}
Cryogenic bypass diodes are part of the baseline powering layout for the circuits of the new $\mathrm{Nb}_{3} \mathrm{Sn}$ based final focus magnets of the high luminosity Large Hadron Collider. They will protect the magnets against excessive transient voltages during a nonuniform quenching process. The diodes are located inside an extension to the magnet cryostat, operated in superfluid helium and exposed to ionizing radiation. Therefore, the radiation tolerance of different types of diodes has been tested at cryogenic temperatures in CERN's CHARM irradiation test facility during its 2018 run. The forward bias characteristics, the turn-on voltage and the reverse blocking voltage of each diode were measured weekly at $4.2 \mathrm{~K}$ and $77 \mathrm{~K}$, as a function of the accumulated radiation dose. The diodes were submitted to a total dose close to $12 \mathrm{kGy}$ and a $1 \mathrm{MeV}$ neutron equivalent fluence of $2.2 \times 10^{14} \mathrm{~cm}^{-2}$. After the end of the irradiation program the annealing behavior of the diodes was tested by increasing the temperature slowly to $293 \mathrm{~K}$. This paper describes the experimental setup, the measurement procedure and the analysis of the measurements performed during the irradiation program as well as the results of the annealing study.
\end{abstract}

DOI: 10.1103/PhysRevAccelBeams.23.053502

\section{INTRODUCTION}

CERN's Large Hadron Collider (LHC) and its upgrade high-luminosity LHC (HL-LHC) rely on superconducting magnets to achieve high magnetic fields and large field gradients, which are necessary to guide high energy hadrons on a circular trajectory and to focus the charged particle beams in four interaction points where two counterrotating beams collide. The LHC main dipole and quadrupole circuits located in each of its eight arcs are powered in long strings of magnets with a length of about $2.8 \mathrm{~km}$. Each of the 8 main dipole and 16 main quadrupole circuits are powered by a single $13 \mathrm{kA}$ power converter. In case one magnet within such a circuit transitions from the

\footnotetext{
*andreas.will@cern.ch
}

Published by the American Physical Society under the terms of the Creative Commons Attribution 4.0 International license. Further distribution of this work must maintain attribution to the author(s) and the published article's title, journal citation, and DOI. superconducting to the normal conducting state, i.e., experiences a quench, the current in the circuit needs to be bypassed around the quenched magnet. This is achieved by connecting a bypass diode in parallel to each magnet. This diode starts to conduct as soon as the resistive voltage of the quenched magnet is higher than its turn-on voltage.

The diodes in the LHC main dipole and quadrupole magnets are mounted in proximity to the magnets allowing short, local routing of the current bus bars. However, due to their proximity to the beam axis, the diodes are impacted by secondary particle debris from interaction of the beams with residual gas molecules or by collision debris from the interaction region. Therefore, the assessment of the radiation tolerance of such diodes is of high importance, to ensure that the operational parameters stay within the specification during the lifetime of the circuit.

The new HL-LHC final focus quadrupole magnets, called the inner triplets, will be installed in the high luminosity interaction regions located at IR1 (ATLAS) and IR5 (CMS) [1]. They will be based on $\mathrm{Nb}_{3} \mathrm{Sn}$ superconducting coils [1-4]. Cryogenic diodes are foreseen in 
the baseline of the powering scheme of these new inner triplets. The diodes will be exposed to large radiation dose and particle fluences due to their location in proximity of the interaction points. The expected radiation levels in these cold diodes will exceed the ones of the existing LHC cold diodes by one order of magnitude. Therefore, based on the experience of the LHC cold diode testing, an irradiation test program was launched to qualify two new prototype diodes and to determine a diode type suitable for HL-LHC. The LHC type diodes were as well included in this program to verify their suitability for the HL-LHC era.

This paper describes the experimental setup of the irradiation program and its results.

\section{A. Radiation tolerance of LHC type diodes}

For LHC's main lattice superconducting magnets, a suitable diode type has been found and qualified in several test programs [5-7]. The LHC cold diodes are diffusion type power diodes with a n-base width of $10 \mu \mathrm{m}$ [5], mounted in a small extension of the magnet helium vessel and have been successfully used during the nearly ten years of LHC operation.

The expected total ionizing dose (TID) $\Phi_{D}$ and the $1 \mathrm{MeV}$ neutron equivalent (neq) fluence $\Phi_{\text {neq }}$ for the diodes of the LHC dipoles over the machine lifetime was estimated to be $\Phi_{D}=200 \mathrm{~Gy}$ and $\Phi_{\text {neq }}=1.2 \times 10^{12} \mathrm{~cm}^{-2}[6]$. Extensive studies have been performed, exposing these diodes to $\Phi_{D}=2 \mathrm{kGy}$ and $\Phi_{\text {neq }}=3 \times 10^{13} \mathrm{~cm}^{-2}$ [7] and evaluating the behavior under the influence of radiation, at cryogenic temperatures. The same type of diode had later been qualified for the FAIR project, to $\Phi_{D}=1.2 \mathrm{kGy}$ and $\Phi_{\text {neq }}=2.2 \times 10^{14}$ [8]. However, the irradiation was performed at $77 \mathrm{~K}$ and some of the tested diodes were warmed up to room temperature after each irradiation step.
Furthermore, testing was only done up to a maximum current of $7.5 \mathrm{kA}$.

Two dipole diodes that had been installed in the LHC for the past 10 years of operation have been extracted recently during the long shutdown (LS2). They were extracted from a position were the lifetime dose was expected to be maximal along the LHC ring and had seen up to 500 Gy since their installation. Further studies on these diodes are foreseen in the near future [9].

\section{B. HL-LHC inner triplet circuits}

Figure 1 shows the electrical layout of the HL-LHC inner triplet circuits in IR1 and IR5. Cold diodes will be installed in parallel to the quadrupole magnets Q1, Q2a, Q2b, and Q3. The quench protection system of these circuits is designed to quench all magnets in case of one circuit element changes into its normal conducting state. Therefore, these diodes will protect the magnets against excessive transient voltages during a nonuniform quenching process.

The diodes will be located in an extension to the cryostat close to the separation dipole D1 and immersed in superfluid helium. They will be exposed to radiation induced by the collision debris from the interaction point. A dose of $\Phi_{D}=12 \mathrm{kGy}$ and a fluence $\Phi_{\text {neq }}=5 \times 10^{13} \mathrm{~cm}^{-2}$ was estimated, using the FLUKA Monte Carlo code [12,13], at the foreseen position of diodes for the lifetime of the HLLHC $[14,15]$. The operating conditions as well as the electrical requirements for the diodes in the circuit shown in Fig. 1, are listed in Table I.

\section{Irradiation program}

An irradiation program in the CERN's High energy Accelerator Mixed field facility (CHARM) [16-18] was performed to validate different types of cold diodes for the

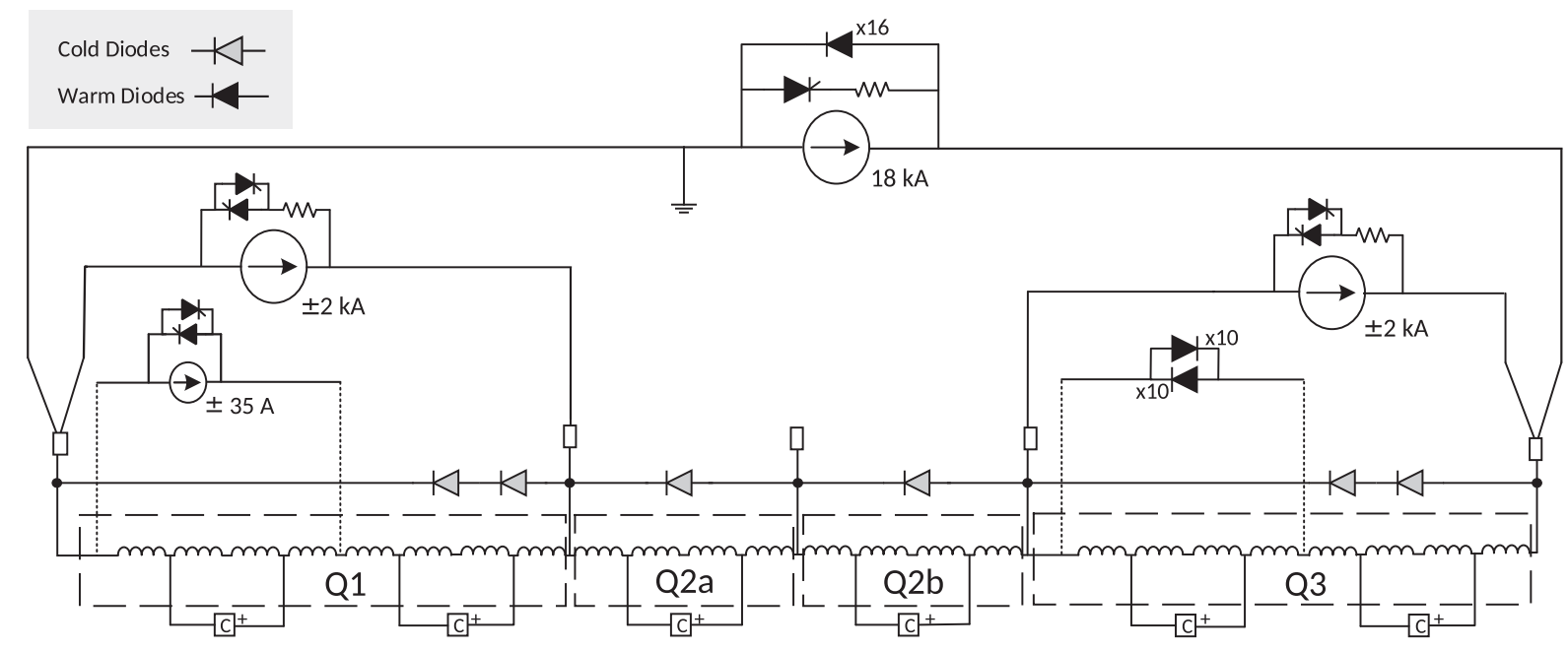

FIG. 1. Circuit layout of the HL-LHC inner triplets for IP1 and IP5. The drawing shows the quadrupoles Q1, Q2a, Q2b, Q3, the main, and trim power converters with their crowbars, the Coupling Loss Induced Quench protection systems (C) [10] and the cold diodes [11]. Image adapted by courtesy of CERN's Magnet Circuit Forum (MCF). 
TABLE I. Operational conditions and electrical requirements of the cryogenic bypass diodes for the HL-LHC inner triplet circuit.

Operational conditions

Operating temperature

Heat pulse integral

Lifetime dose $\left(\Phi_{D}\right)$

Lifetime $1 \mathrm{MeV}$ neq fluence $\left(\Phi_{\text {neq }}\right)$

Maximum current

$1.9 \mathrm{~K}$

$4.9 \times 10^{6} \mathrm{~A}^{2} \mathrm{~s}$ $12 \mathrm{kGy}$

$5 \times 10^{13} \mathrm{~cm}^{-2}$

$7 \mathrm{kA}$

Electrical requirements

Turn-on voltage at $1.9 \mathrm{~K}\left(U_{\text {to }}\right)$

Turn-on time

Diode series resistance at max. current $\left(R_{S}\right)$

Reverse voltage at $1.9 \mathrm{~K}$ for $\mathrm{I}_{\mathrm{rev}}=1 \mathrm{~mA}\left(U_{\text {rev }}\right)$

use in the described radiation environment. The diode properties were characterized in-situ as a function of the integrated dose and particle fluence at cryogenic temperatures. A more detailed description of the irradiation program and its goals has been published in [19].

\section{Experimental setup}

A photo of the inner part of the experimental setup is shown in Fig. 2. The cryostat houses a cryocooler with two stage plate interfaces. One stack of four diodes is attached to each stage. The first stack is thermally anchored on the first stage of the cryocooler, which is operated between $50 \mathrm{~K}$ and $77 \mathrm{~K}$. The second stack is thermally anchored on the second stage of the cryocooler and kept at $\sim 4.2 \mathrm{~K}$. The setup allows to measure the forward and reverse bias characteristics of the diodes. At the first stage, forward current pulses of few millisecond duration and with a maximum current of $18 \mathrm{kA}$ can be applied. To suppress the inductive component in the measured voltage signal, the voltage was measured at the peak of each current pulse, where $\mathrm{d} I / \mathrm{d} t=0$. The forward characteristics was probed step-wise by increasing the current pulse amplitude. The turn-on voltage $U_{\text {to }}$ is measured by applying linear voltage ramps with a ramp rate of 50 and $100 \mathrm{~V} / \mathrm{s}$, while measuring the current flowing through the diode. Once the diode is conducting, the current rises steeply and $U_{\text {to }}$ is measured, when the current reaches a threshold level of $200 \mathrm{~mA}$. On the second stage, the forward current is limited to about $1 \mathrm{~A}$, as self-heating at low temperatures is significant. This limits the measurements under forward bias only to the turn-on voltage $U_{\text {to }}$ on this stage, while on the first stage $U_{\text {to }}$ and the forward voltage $U_{f}$ can be determined for various current amplitudes. The reverse bias voltage $U_{\text {rev }}$ can be measured in-situ for the diodes in both stacks. A logarithmic current sweep from $1 \mu \mathrm{A}$ to $1 \mathrm{~mA}$ is performed and the voltage is recorded. At a reverse current of $1 \mathrm{~mA}$, reverse breakdown occurs, however, the current limit prevents the wafer from damage. A more detailed description of the in-situ measurements can be found in [19].

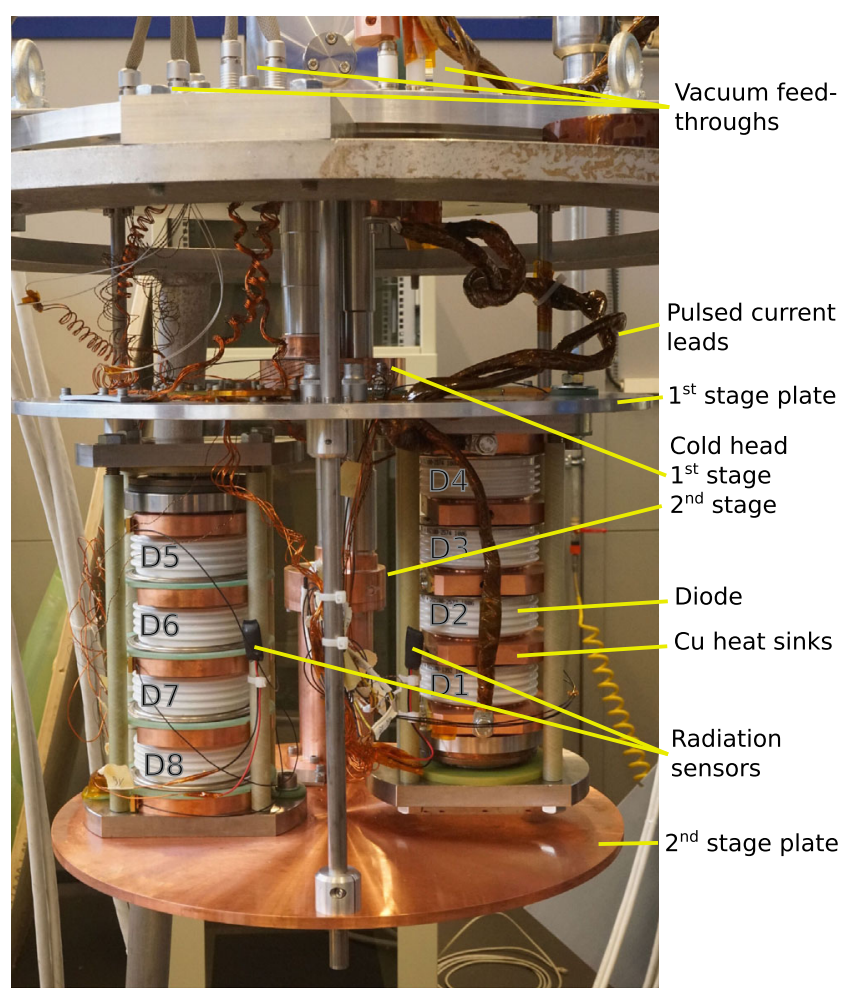

FIG. 2. Inner part of the experimental cryostat for testing of the radiation tolerance of cold diodes for the HL-LHC triplets. The setup is based on a cryocooler and houses two stacks of 4 diodes each. Diodes D1-D4 are mounted on the first stage plate $(77 \mathrm{~K})$, diodes D5-D8 on the second stage plate $(4.2 \mathrm{~K})$.

\section{E. Types of tested diodes}

The diode types used are prototypes of power diffusion diodes, each having a specific $p^{+} n n^{+}$doping profile and a different $n$-base width. The $n$-base width is defined as the distance of the junction depths, i.e., the intersections of the $p^{+}$and $n^{+}$doping profiles with the $n$-doping level of the Silicon wafer before the diffusion process of $\approx 9 \times 10^{13} \mathrm{~cm}^{-3}$. A negative base width suggests that the two profiles overlap. The difference in $n$-base width does neither affect $U_{\text {to }}$ nor $U_{f}(I)$ of these virgin diffusion type diodes, while $U_{\text {rev }}$ decreases with decreasing $n$-base width.

Table II provides an overview of the parameters of the different diodes used in the experiment. The diodes are

TABLE II. Parameters of diode prototypes and their corresponding labels as used in this article. The temperatures at which the in-situ characterization was performed is indicated in brackets.

\begin{tabular}{llcl}
\hline \hline Diode label & & n-base width & $\mathrm{U}_{\text {rev }}$ \\
\hline LHC Reference & $\mathrm{D} 1(77 \mathrm{~K}), \mathrm{D} 8(4.2 \mathrm{~K})$ & $10 \pm 5 \mu \mathrm{m}$ & $520 \pm 10 \mathrm{~V}$ \\
Thin base & $\mathrm{D} 2(77 \mathrm{~K}), \mathrm{D} 7(4.2 \mathrm{~K})$ & $0 \pm 5 \mu \mathrm{m}$ & $400 \pm 5 \mathrm{~V}$ \\
Very thin base & $\mathrm{D} 3(77 \mathrm{~K}), \mathrm{D} 6(4.2 \mathrm{~K})$ & $-10 \pm 5 \mu \mathrm{m}$ & $250 \pm 20 \mathrm{~V}$ \\
& $\mathrm{D} 4(77 \mathrm{~K}), \mathrm{D} 5(4.2 \mathrm{~K})$ & & \\
\hline \hline
\end{tabular}


TABLE III. $\Phi_{D}$ and $\Phi_{\text {neq }}$ for each diode at the end of the irradiation program.

\begin{tabular}{lrrrr}
\hline \hline Diode & D1 & D2 & \multicolumn{1}{c}{ D3 } & D4 \\
\hline$\Phi_{\text {neq }}$ in $10^{14} \mathrm{~cm}^{-2}$ & 2.09 & 2.15 & 2.27 & 2.28 \\
$\Phi_{D}$ in $\mathrm{kGy}$ & 10.40 & 11.17 & 11.06 & 10.24 \\
\hline \hline Diode & $\mathrm{D} 5$ & $\mathrm{D} 6$ & $\mathrm{D} 7$ & $\mathrm{D} 8$ \\
\hline$\Phi_{\text {neq }}$ in $10^{14} \mathrm{~cm}^{-2}$ & 1.74 & 1.75 & 1.70 & 1.66 \\
$\Phi_{D}$ in $\mathrm{kGy}$ & 11.00 & 11.02 & 12.20 & 9.75 \\
\hline \hline
\end{tabular}

named $L H C$ reference (D1, D8), thin base (D2, D7), and very thin base (D4, D5, D6, D7). The $L H C$ reference diode is the same type as currently installed across the main dipole and quadrupole magnets of the LHC. Thin and very thin base types are new prototypes, especially manufactured for this irradiation program.

\section{F. Dosimetry}

Table III summarizes the total ionizing dose $\left(\Phi_{D}\right)$ and fluence $\left(\Phi_{\text {neq }}\right)$ accumulated in each diode at the end of the 6 months long irradiation program. The simulated dose and fluence rates were validated using two PIN diodes mounted at the diode stacks and two RADMON detectors [20] on the cryostat walls upstream and downstream in respect to the beam during a calibration run prior to the irradiation program. Based on these results, FLUKA simulations were performed to derive $\Phi_{D}$ and $\Phi_{\text {neq }}$ for each diode wafer as a function of the number of protons impacting on the CHARM target. The difference between the diodes presented in Table III can be explained by their geometrically different position with respect to the beam axis. From the calibration run an uncertainty of $25 \%$ was estimated on the absolute values given in Table III. This includes the uncertainty of the cross check between RADMON measurements and FLUKA simulations, as well as the uncertainty of the protons on target.

\section{RESULTS OF THE IRRADIATION PROGRAM}

This section presents the results of the measurements. Where possible, all prototypes are shown for comparison. Some data is only shown for the LHC reference and very thin base diodes, as the thin base diodes usually rank in between the two other types in terms of their sensitivity to radiation.

\section{A. Behavior of forward voltage due to radiation damage measured at $77 \mathrm{~K}$}

Figure 3 shows the relative increase of the measured forward voltage $U_{f}$ of diode D1 and D4 as a function of $\Phi_{\text {neq }}$ for currents from $1 \mathrm{kA}$ to $18 \mathrm{kA}$. The absolute value of $U_{f}$ for the virgin diodes varied from $1.14 \mathrm{~V}$ at $1 \mathrm{kA}$ to
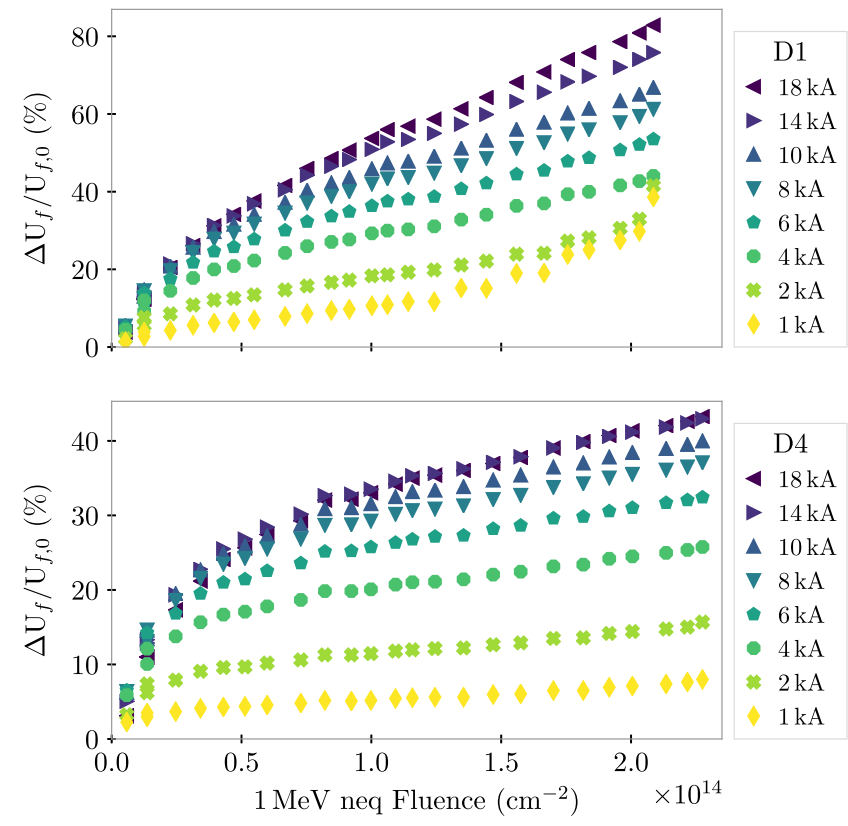

FIG. 3. Measured relative increase of the forward voltage $U_{f}$ in diode D1 (top) and diode D4 (bottom, as in [21]) as function of $\Phi_{\text {neq }}$, for currents from $1 \mathrm{kA}$ to $18 \mathrm{kA}$.

$1.72 \mathrm{~V}$ at $18 \mathrm{kA}$. A steep increase of the forward voltage up to $\Phi_{\text {neq }} \sim 0.75 \times 10^{14} \mathrm{~cm}^{-2}$ can be observed for currents above $2 \mathrm{kA}$. Above $\Phi_{\text {neq }} \sim 0.75 \times 10^{14} \mathrm{~cm}^{-2}$, the original steep increase flattens out and from $\Phi_{\text {neq }} \sim 1 \times 10^{14} \mathrm{~cm}^{-2}$, the increase appears to become linear. The relative increase at $18 \mathrm{kA}$ reaches $\sim 43 \%$ for diode D4 and $\sim 83 \%$ for diode D1 at the end of the irradiation program. All diodes showed qualitatively the same behavior but with significant differences in the slope of the linear part. For currents $\lesssim 2 \mathrm{kA}$, diode D1 shows a nonlinear increase for $\Phi_{\text {neq }} \gtrsim 1 \times 10^{14} \mathrm{~cm}^{-2}$.

Figure 4 shows the relative change of $U_{f}$ versus $\Phi_{\text {neq }}$ at $18 \mathrm{kA}$ for the diodes D1, D2, D3, and D4. The diodes with the smallest $n$-base widths (D3, D4) showed the lowest increase of $U_{f}$ of about $40 \%$ and behave very similar. For the LHC reference diode (D1) $U_{f}$ nearly doubles and the thin base width diode (D2) lies in between the two other types. The lower degradation of the forward voltage correlates-as expected — with the reduction of base width.

\section{B. Temperature increase in Si-wafer}

The forward characteristics was probed stepwise by increasing the current pulse amplitude. All current pulses have been applied at a starting temperature of $77 \pm 0.5 \mathrm{~K}$. The current pulses have a duration of few milliseconds, with the current peak reached after about $700 \mu$ s. The energy deposited into the wafer due to the current is nonnegligible. It increases with increasing amplitude and raises the temperature of the wafer. This effect causes a decrease of the measured voltage signal, due to the negative 


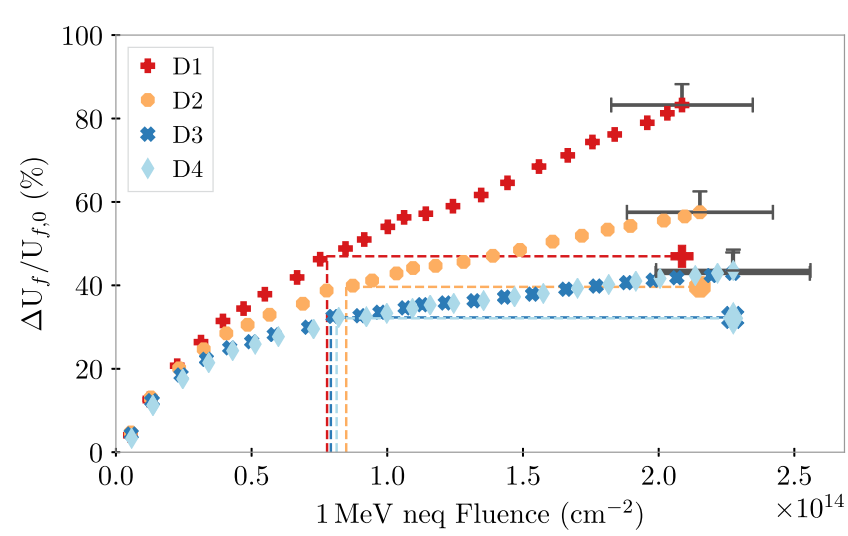

FIG. 4. Relative change of the forward voltage $U_{f}$ versus $\Phi_{\text {neq }}$ at $18 \mathrm{kA}$ for the diodes D1, D2, D3, and D4. The last measurement points of each diode indicate the value of $U_{f}$ after a thermal cycle to room temperature. The dotted lines indicate the equivalent $\Phi_{\text {neq }}$ levels of $U_{f}$ after the thermal cycle. The errorbars correspond to a $25 \%$ uncertainty in $\Phi_{\text {neq }}$ and an error of $+8 \%$ in the forward voltage due to self-heating. Image adapted from [21].

temperature dependence of the forward voltage. The wafer temperature during a current pulse was evaluated with a transient thermal simulation of the experimental setup using the measured forward characteristic. The simulations yielded wafer temperatures of $\sim 84 \mathrm{~K}$ for $\mathrm{D} 4$ and $\sim 87 \mathrm{~K}$ for D1 when reaching the peak of $18 \mathrm{kA}$ for their characteristics at the end of the irradiation program. The measurements during the thermal cycle allowed to derive $d U_{f} / d T$. The calculated temperature increase is, therefore, equivalent to a decrease of $U_{f}$ of about $200 \mathrm{mV}$. This corresponds to $8 \%$ of the measured voltage signal at this current level. For simplicity, this effect was considered as an absolute systematic error, indicated by the error-bars in Fig. 4. Note, that the self-heating effect is negligible for currents below $12 \mathrm{kA}$.

\section{Dynamic series resistance increase}

The dynamic series resistance of the diodes was calculated numerically from the gradient of the measured forward characteristic $U_{f}\left(I_{f}\right)$. The values are shown in Fig. 5 as a function of the current for diode D1, D2, and D4. The increase in resistance with increasing $\Phi_{\text {neq }}$ is less than $0.12 \mathrm{~m} \Omega$ for all diodes. The specified upper limit of $0.5 \mathrm{~m} \Omega$, as mentioned in Table I, was not exceeded in any case.

\section{Fits using the full Shockley equation}

The Shockley equation [22] defines the voltage drop $U_{f}$ over a diode, when a current $I$ is flowing, as

$$
U_{f}=I R_{s}+\frac{l k_{B} T}{e} \ln \left(\frac{I}{I_{s}}+1\right) .
$$

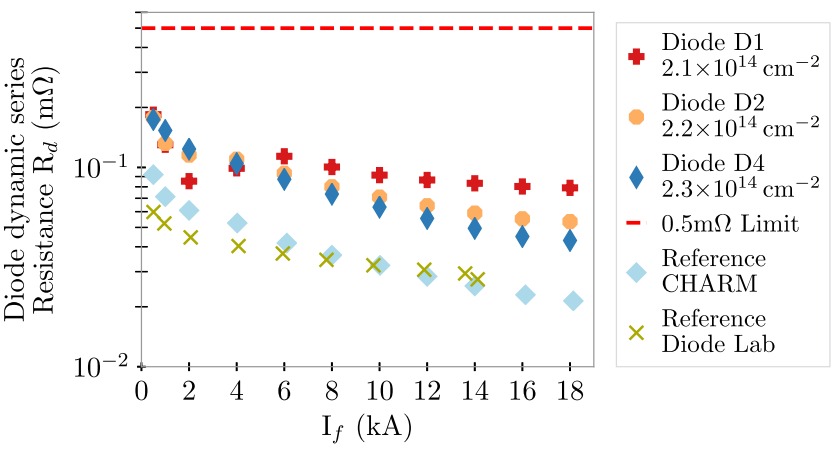

FIG. 5. Dynamic series resistance $R_{d}$ of diodes D1, D2, and D4 as a function of the current after the end of the irradiation program. The series resistance was calculated from the gradient of the measured forward characteristics curves. The reference values were derived from the CHARM calibration run before irradiation and from a lab setup.

Here, $k_{B}$ is the Boltzman constant, $l$ the ideality factor, $e$ the elementary charge and $I_{s}$ the saturation current. The series resistance $R_{s}$ was extracted as the tangent's slope on the measured $U_{f}\left(I_{f}\right)$ curves for the peak of the current pulse. Figure 6 shows the increase of the series resistance as a function of $\Phi_{\text {neq }}$ for diodes D1 to D4. The series resistivity resulting from the ohmic resistance of the n-base silicon can be expressed as

$$
\rho=\frac{1}{\frac{e^{2}}{m_{e}} n \tau_{e}+\frac{e^{2}}{m_{h}} p \tau_{h}},
$$

with the scattering lifetimes of electrons and holes $\tau_{e / h}$, electron and hole effective masses $m_{e / h}$ and the charge densities of electrons and holes $n$ and $p$ [23]. The inverse lifetimes grow linearly with increasing

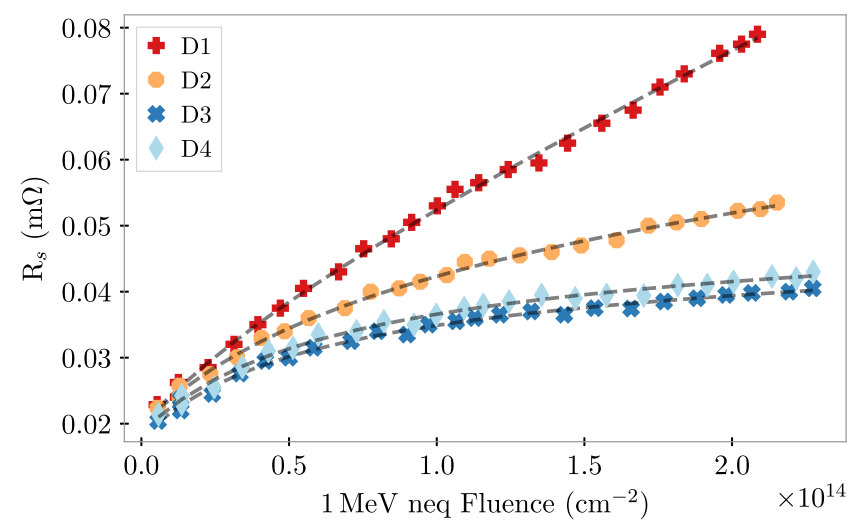

FIG. 6. Schockley series resistance extracted from the forward characteristics of the diodes, as a function of $\Phi_{\text {neq }}$. A fit using a functional dependence in the form of Eq. (2) was performed. The fit parameters for D1 are $K_{R, h}=9.8 \times 10^{-19}$ and $K_{R, e}=$ $2.7 \times 10^{-19}$, for D4 the fit yields $K_{R, h}=7.7 \times 10^{-21}$ and $K_{R, e}=$ $1.2 \times 10^{-18}$ in the units of $\Omega \mathrm{cm}^{2}$ respectively. 
radiation displacement damage and, therefore, in the first order are proportional to $\Phi_{\text {neq }}[24]$ :

$$
\frac{1}{\tau_{e / h}}=\frac{1}{\tau_{0, e / h}}+K_{\tau} \Phi_{\text {neq }}
$$

with proportionality factor $K_{\tau}$. Inserting Eq. (3) in Eq. (2), the resistivity can be derived as a function of $\Phi_{\text {neq. }}$. The main contribution to the resistance originates from the low doped narrow $n$-base region, where the resistivity is highest. As an approximation, the doping profiles are assumed to be step-functions, and the resistance is derived as

$R_{s}=\frac{1}{\left(R_{0, e}+K_{R, e} \Phi_{\text {neq }}\right)^{-1}+\left(R_{0, h}+K_{R, h} \Phi_{\text {neq }}\right)^{-1}}$,

with the degradation coefficients $K_{R, h}$ and $K_{R, e}$ in units of $\Omega \mathrm{cm}^{2}$. A fit to the measured series resistances, using the above equation, results in the values of $K_{R, e}$ and $K_{R, h}$ as indicated in Fig. 6. Due to the different effective mass of electrons and holes, the contribution of the two lifetimes differs significantly, leading to different orders of magnitude for the degradation coefficients. As long as one of the two lifetimes is dominating, a linear growth of resistance can be assumed [see Eq. (2)]. This is the case in the regime $\Phi_{\text {neq }} \gtrsim 1 \times 10^{14} \mathrm{~cm}^{-2}$, where the growth becomes linear (compare [6]).

The Schockley equation shown in Eq. (1) with the dependencies in Eq. (4) has seven free fit parameters. However, the resistance as a function of fluence, presented in Eq. (4), can be extracted directly from the measured data, as described above. Three free fit parameters remain: the ideality factor $l$ and the saturation current $I_{s}$, for which it is usually assumed [25]

$$
I_{s}\left(\Phi_{\text {neq }}\right)=I_{s, 0}-K_{I} \Phi_{\text {neq }}
$$

with original saturation current $I_{s, 0}$ and the damage coefficient $K_{I}$. The fits on the full dataset $U_{f}\left(I_{f}, \Phi_{\text {neq }}\right.$, $T=77 \mathrm{~K}$ ) using this model are shown in Fig. 7 for D1 and D4. The model represents the trends of the measurement very well. The onset of nonlinear growth of voltage in the lower current regime $\lesssim 2 \mathrm{kA}$ can be clearly seen. It results mathematically from the decrease in $I_{s}$, leading to a divergence in the logarithmic part of Eq. (1). The linear decrease as in Eq. (5) can therefore only be valid until $I_{s}$ approaches zero. Close to zero, the linear model is no longer physical but approaches zero asymptotically. Within the measured range of $\Phi_{\text {neq }} \sim 2 \times 10^{14} \mathrm{~cm}^{-2}$, the linear model stays valid.

To avoid the nonphysical divergence for $I_{s} \rightarrow 0$, an exponentially decaying saturation current model is proposed

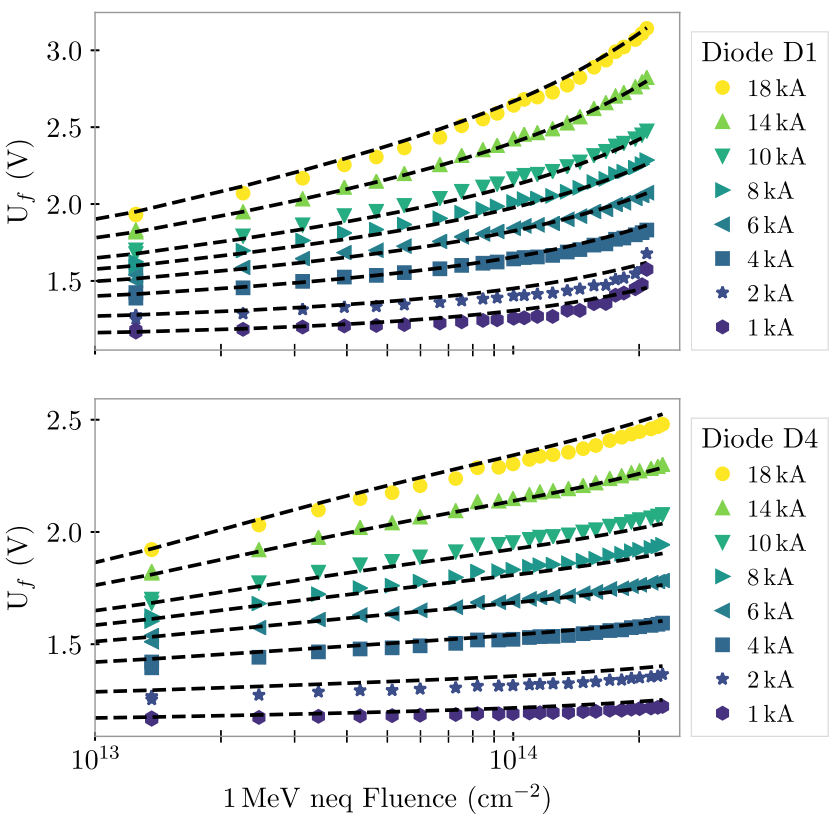

FIG. 7. Schockley equation fit on the full dataset $U_{f}\left(I_{f}, \Phi_{\text {neq }}, T=77 \mathrm{~K}\right)$ for diodes D1 and D4.

$$
I_{s}\left(\Phi_{\text {neq }}\right)=I_{s, 0} \cdot \exp \left(-K_{I} \Phi_{\text {neq }}\right),
$$

which can be used for extrapolation. For low regimes of damage, i.e., $K_{I} \Phi_{\text {neq }} \ll 1$, this can be expanded in a Taylor series yielding

$$
I_{s}\left(\Phi_{\text {neq }}\right) \approx I_{s, 0}-K_{I, 1} \Phi_{\text {neq }}+\mathcal{O}\left(K_{I, 2} \Phi_{\text {neq }}\right)^{2},
$$

and representing Eq. (5) in the first order approximation.

The fits, including the extrapolation for both the exponential and linear models are plotted in Fig. 8. They clearly show the divergence of the linear model. For diode D1, this starts shortly after the maximum measured fluence. For the other diodes, the linear and exponential models coincide for a longer range, before the divergence in the linear model

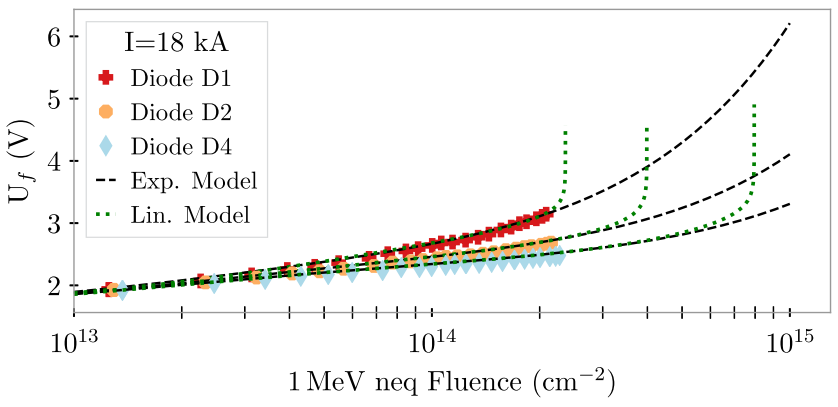

FIG. 8. Schockley equation fit on the full datasets $U_{f}\left(I_{f}, \Phi_{\text {neq }}, T=77 \mathrm{~K}\right)$ for diodes D1, D2, and D4, based on the exponential model. The forward voltage at a current of $18 \mathrm{kA}$ was extrapolated up to a $\Phi_{\text {neq }}=1 \times 10^{15} \mathrm{~cm}^{-2}$ assuming the linear and exponential model. 

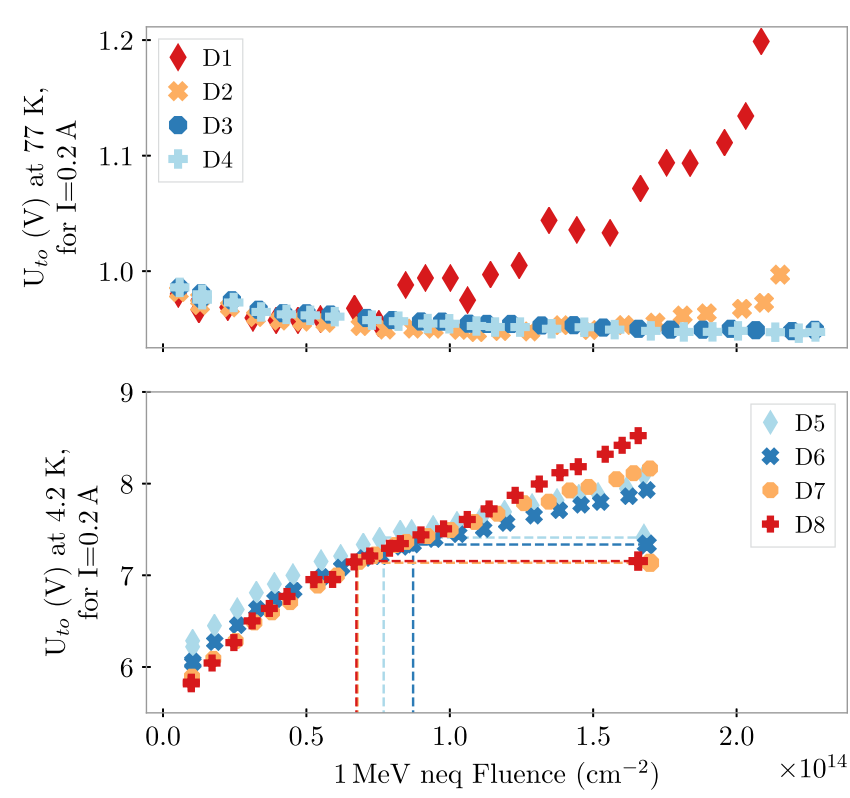

FIG. 9. Top: Turn-on voltage $U_{\text {to }}$ of D1, D2, D3, and D4 as a function of $\Phi_{\text {neq }}$ measured at a current of $I=0.2$ A at $77 \mathrm{~K}$ [21]. Bottom: Turn-on voltage $U_{\text {to }}$ of D5, D6, D7, and D8 as a function of $\Phi_{\text {neq }}$ measured at a current of $I=0.2 \mathrm{~A}$ at $4.2 \mathrm{~K}$. The last measurement points of each diode indicate the value of $U_{\text {to }}$ after a thermal cycle to room temperature. The dotted lines indicate the equivalent $\Phi_{\text {neq }}$ levels for these annealed $U_{\text {to }}$ values.

separates the two curves abruptly. For this regime the linear model loses validity and the exponential model should be used. Future measurements have to show if the proposed model is valid for those fluences.

\section{E. Turn-on voltage}

The turn-on behavior was studied in this experiment by applying a linear voltage ramp of $50 \mathrm{~V} / \mathrm{s}$ and $100 \mathrm{~V} / \mathrm{s}$ and measuring the current through the diode. The turn-on voltage $U_{\text {to }}(I)$ is then defined as the voltage at a current of $200 \mathrm{~mA}$. This definition is arbitrary, but quite robust as the current rises very steeply once the diode is conducting, and a change in this criterion of current level does not significantly alter the observed turn-on voltages. The criterion ensures that the diodes are in a fully conducting state. Figure 9 shows $U_{\text {to }}$ for all diodes as a function of $\Phi_{\text {neq. }} . U_{\text {to }}$ increased by $1.8 \mathrm{~V}(30 \%)$ for diode D5 and by $2.7 \mathrm{~V}(46 \%)$ for diode D8. Similar to the observation for the change of $U_{f}$, the $L H C$ reference diodes experience the strongest and the very thin base diodes the least degradation.

The measured turn-on voltage at $77 \mathrm{~K}$ does not significantly alter after exposure to $\Phi_{\text {neq }}$ for D2, D3, and D4, as shown in Fig. 9. D1 (LHC reference) shows an irregular increase for $\Phi_{\text {neq }} \gtrsim 1 \times 10^{14} \mathrm{~cm}^{-2}$. This corresponds to the nonlinear increase of the forward bias voltage at lower currents $(\lesssim 2 \mathrm{kA})$ in the LHC reference diodes mentioned above.

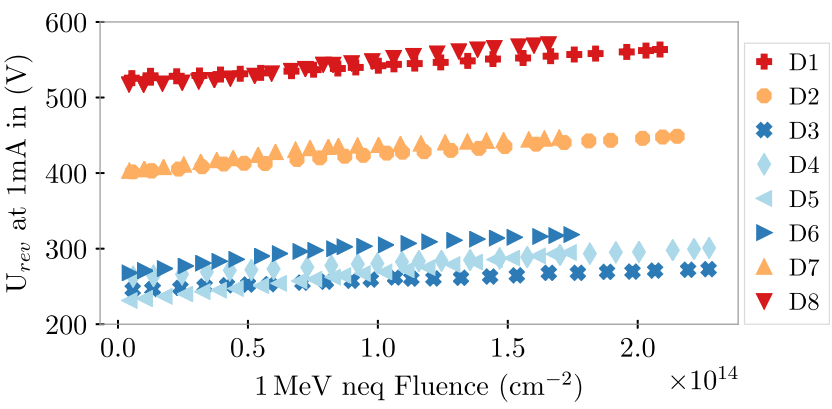

FIG. 10. Reverse blocking voltage $U_{\text {rev }}$ at a reverse current of $I_{\text {rev }}=1 \mathrm{~mA}$, as a function of $\Phi_{\text {neq }}[21]$.

\section{F. Reverse blocking voltage}

The leakage current under reverse bias is measured as a function of the applied voltage up to the reverse breakdown. In this study a logarithmic current sweep was performed from $1 \mu \mathrm{A}$ to $1 \mathrm{~mA}$ and the voltage for each current step was measured. The reverse blocking voltage $U_{\text {rev }}$ was defined as the voltage at $I_{\mathrm{rev}}=1 \mathrm{~mA}$. Figure 10 shows the evolution of the measured $U_{\text {rev }}$ for all diodes as a function of $\Phi_{\text {neq. }}$. An increase of up to $20 \%$ was observed. It is important to mention that diodes of the same type have a very similar behavior of $U_{\text {rev }}$, independent of their operating temperature $(4.2 \mathrm{~K}$ or $77 \mathrm{~K})$.

\section{TEMPERATURE DEPENDENCE AND ANNEALING}

At the end of the irradiation program, an annealing study was performed, where the diodes were warmed up to $293 \mathrm{~K}$ and temperature dependent measurements were performed on various temperature levels during this warm-up. In a subsequent cooldown, the diodes' properties were measured and compared to the values before the thermal cycle.

In HL-LHC, two scenarios of warm-up are envisaged. First, during a quench, where the diodes could reach roomtemperature for a short period of time. Second, during long shutdowns of the accelerator a warm up for weeks or months could be expected. These long warm-ups could be expected twice during the lifetime of the cold diodes for the new HL-LHC triplet circuits. Therefore, it is important to understand the impact of such warm-ups on the diode properties.

\section{A. Annealing of $\boldsymbol{U}_{f}$ and $\boldsymbol{U}_{\text {to }}$}

The annealing effect due to the thermal cycle is clearly visible in the measured turn-on $\left(U_{\mathrm{to}}\right)$ and forward $\left(U_{f}\right)$ voltages, as indicated in Figs. 4 and 9. Figure 4 shows the values of forward voltage after annealing and indicates the equivalent level of $\Phi_{\text {neq }}$. The absolute voltage change after annealing is different for all diode types. However, the equivalent $\Phi_{\text {neq }}$ levels are very similar. This agrees with the expectations as annealing is a thermally driven process and proportional to the defect density. 


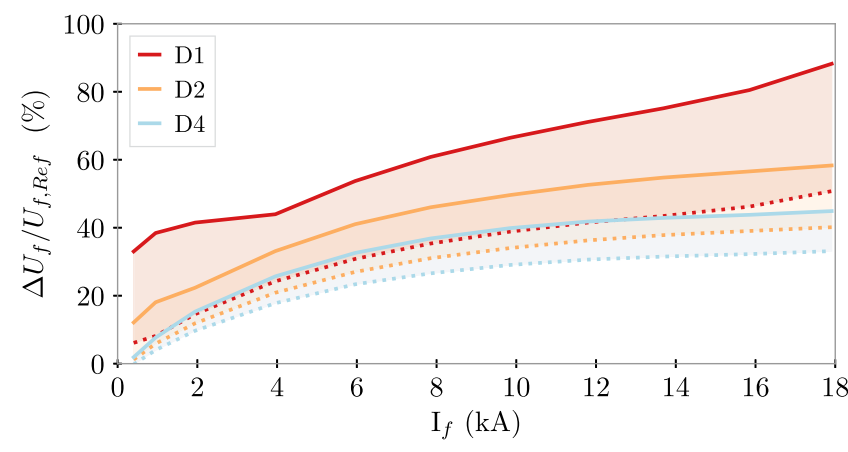

FIG. 11. Relative increase of the diodes' forward voltages at the end of the irradiation program (solid lines) and after the thermal cycle (dotted lines), as a function of the current. The measurements were performed at $77 \mathrm{~K}$. D3 was omitted in this plot for better visibility, however it behaves very similar to D4.

The annealing as a function of the current is shown in Fig. 11 for diodes D1, D2, and D4. The measured relative change of $U_{f}$ at the end of the irradiation program and after the thermal cycle are plotted for all currents. The nonlinear increase in forward voltage at lower currents for D1, visible as a bump on the curve, disappeared after the thermal cycle. The reason for this behavior is unknown.

\section{B. Temperature dependence of $U_{\text {to }}$}

The temperature dependence of $U_{\text {to }}$ for diode D5 is shown in Fig. 12 as an example. The measurements were performed from $3.9 \mathrm{~K}$ to room temperature during a slow warm-up following the end of the irradiation program.

$U_{\text {to }}$ is almost constant between $3.9 \mathrm{~K}$ and $5 \mathrm{~K}$. Therefore, no significant increase of $U_{\text {to }}$ is expected between $3.9 \mathrm{~K}$ and the operational temperature of the cold diodes in HLLHC of $1.9 \mathrm{~K}$ as indicated in Fig. 12. With increasing temperature $U_{\text {to }}$ decreases rapidly to $\sim 1.2 \mathrm{~V}$ at $30 \mathrm{~K}$ and finally to $\sim 1 \mathrm{~V}$ at $50 \mathrm{~K}$ from where it decreases linearly to a value of $0.3 \mathrm{~V}$ at room temperature. The other diodes in the experiment show a similar behavior.

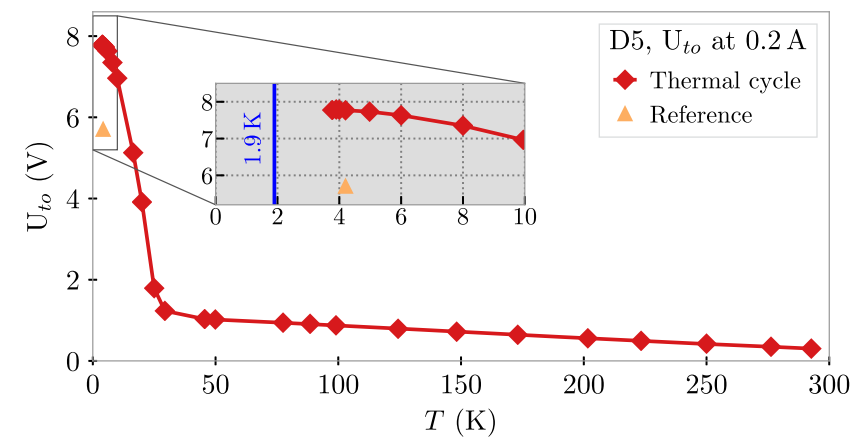

FIG. 12. $U_{\text {to }}$ of diode D5 from $3.9 \mathrm{~K}$ to room temperature and zoom between 0 and $10 \mathrm{~K}$. The orange reference indicated the $U_{\text {to }}$ value at the beginning of the irradiation program [21].

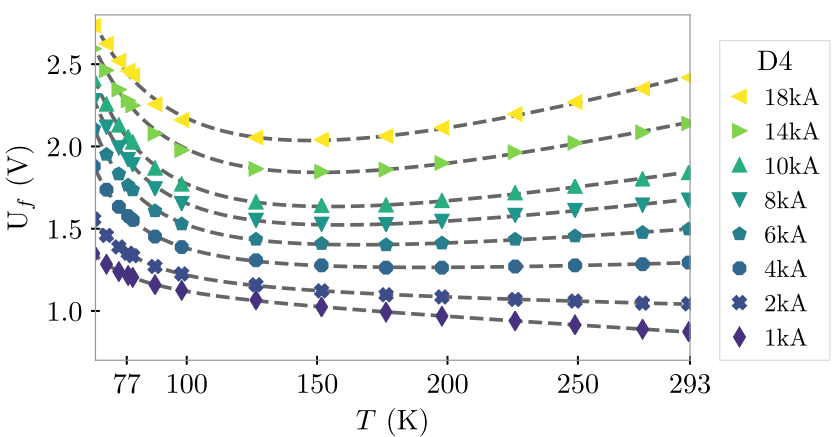

FIG. 13. $U_{f}$ of diode D4 from $65 \mathrm{~K}$ to room temperature for different current levels.

\section{Temperature dependence of $\boldsymbol{U}_{\boldsymbol{f}}$}

Figure 13 shows the dependence of $U_{f}$ on temperature, from $65 \mathrm{~K}$ to room temperature for different current levels for diode D4. Due to the negative temperature coefficient of the diode $U_{f}$ decreases with increasing temperature. For currents $I_{f} \gtrsim 2 \mathrm{kA}$, the influence of the voltage drop over the base resistance becomes significant, causing an increase of $U_{f}$ with increasing temperature for $T \gtrsim 150 \mathrm{~K}$.

The curves are fitted assuming a dependence of the form

$$
U_{f}=C_{1}(I) \cdot \exp \left(\frac{T_{0}}{T}\right)+C_{2}(I) \cdot T+C_{3}(I),
$$

with fit coefficients $C_{1}, C_{2}, C_{3}$, the exponential term originating from the temperature dependence of the base resistance and the linear temperature dependence originating from the Shockley equation (1). The fits reproduce the measurements well and can, e.g., be used for circuit modeling or thermoelectric simulations, when analytical formulations are required. The behavior of the other diodes is similar.

\section{Leakage current under reverse bias}

Figure 14 shows the leakage current $I_{r}$ as a function of the reverse bias voltage $U_{r}$ at selected temperatures for D3. The measurements were performed during the thermal cycle at the end of the irradiation program. The leakage current for a given reverse bias voltage shows a strong temperature dependence between $\sim 250 \mathrm{~K}$ and room temperature. Comparing the room temperature measurements before (red) and after the irradiation (yellow) shows an increase of the leakage current by two orders of magnitude due to the radiation damage.

Based on literature, the leakage current as a function of the reverse bias voltage increases linearly with $\Phi_{\text {neq }}[25]$

$$
\Delta I=K_{I} \cdot V \cdot \Phi_{\text {neq }},
$$

with the damage coefficient $K_{I}$ and the wafer active volume $V$. Assuming $V$ as the full diode wafer volume and using 


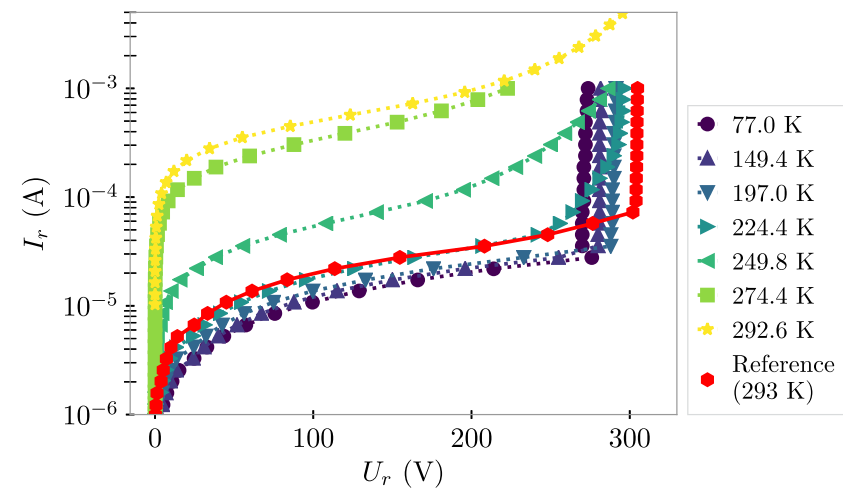

FIG. 14. Leakage current of D3 as a function of the reverse bias voltage at different temperature levels after the irradiation program. The measurements were performed during a thermal cycle at the end of the irradiation program. For reference the leakage current of the same diode at room temperature before the irradiation program is shown (red).

$\Phi_{\text {neq }}$ of D3 at the end of the irradiation program, one can derive from Eq. (9) the damage coefficient as $K_{I}=\left(1.2_{-1.1}^{+3}\right) \times 10^{-17} \mathrm{~A} \mathrm{~cm}^{-1}$. This value is in the same order of magnitude as quoted in literature. Note, that the change of the leakage current at room temperature as function of $\Phi_{\text {neq }}$ has not been measured during the irradiation program, as no intermediate warm-ups were performed. However, the observed increase of the leakage current after the irradiation is fully conform with the requirements for the new HL-LHC triplets.

\section{SUMMARY AND CONCLUSION}

Three types of power diodes with different $p^{+} n n^{+}$ doping profiles, i.e., different $n$-base widths, were irradiated up to $\Phi_{\text {neq }}=2.2 \times 10^{14} \mathrm{~cm}^{-2}$. During the irradiation, the diodes were kept at cryogenic temperatures of either $4.2 \mathrm{~K}$ or $77 \mathrm{~K}$. In-situ measurements of the diode characteristics in forward and reverse bias mode were performed regularly with pulsed currents up to $18 \mathrm{kA}$. The specified operational parameter limits of the diodes (see Table I) for the use in the new HL-LHC triplet circuits were not exceeded for any of the diode types at the end of the irradiation program. The very thin base-width prototypes showed the least degradation in the forward bias voltage at $77 \mathrm{~K}$. No significant difference in the turn-on behavior at $4.2 \mathrm{~K}$ between the different prototypes was observed. The turn-on voltage at $77 \mathrm{~K}$ as well as the forward voltage in the low current regime up to $2 \mathrm{kA}$ of the $\mathrm{LHC}$ reference diode show a nonlinear increase for $\Phi_{\text {neq }} \gtrsim 1 \times 10^{14} \mathrm{~cm}^{-2}$.

An exponential behavior in the decrease of the diodes' saturation current was assumed, to overcome a physically implausible divergence in the Schockley equation, that arises when the saturation current approaches zero. Fits of the Schockley equation on the dataset, using this extension, allow an extrapolation up to $\Phi_{\text {neq }} \sim 1 \times 10^{15} \mathrm{~cm}^{-2}$. At the end of the program, the annealing of the radiation damage was studied during a slow increase of the temperature to room temperature. The annealing of the accumulated radiation damage recovered several tens of percent of the degradation of the forward voltage $U_{f}$, depending on the diode type. Although the absolute amount of annealed voltage is different for all diode types, the equivalent $\Phi_{\text {neq }}$ levels are very similar.

Following the positive outcome of this study, the cryogenic by-pass diodes were implemented into the baseline circuit layout of the new HL-LHC triplets. Furthermore, the very thin base-width type diodes were selected for this application.

\section{ACKNOWLEDGMENTS}

This work was supported by CERN's High Luminosity LHC project and the Wolfgang-Gentner programme of the German Federal Ministry of Education and Research. The authors would like to thank A. Infantino, R. Garcia-Alia, M. Krawina, and C. Cangialosi for the FLUKA simulations of the experimental setup and the subsequent dosimetry in CHARM. Special thanks goes to S. Danzeca, J. Lendaro. and to the colleagues of CERN's Radio Protection and Vacuum groups for their support during the measurement program in the CHARM test facility. Furthermore the authors would like to thank Dynex Semiconductor Ltd. for the development of the new prototype diodes as well as the LHC type diodes. Finally the authors would like to thank the colleagues from the Machine Protection group for their support during the design, construction, test, installation, operation, and removal of the experimental setup.

[1] High-Luminosity Large Hadron Collider (HL-LHC): Technical Design Report V. 0.1 (CERN Yellow Reports: Monographs, Geneva, 2017).

[2] P. Ferracin et al., Magnet design of the $150 \mathrm{~mm}$ aperture low- $\beta$ quadrupoles for the high luminosity LHC, IEEE Trans. Appl. Supercond. 24, 1 (2014).

[3] G. Ambrosio, Nb3Sn high field magnets for the high luminosity LHC upgrade project, IEEE Trans. Appl. Supercond. 25, 1 (2015).

[4] E. Todesco et al., Design studies for the low-beta quadrupoles for the LHC luminosity upgrade, IEEE Trans. Appl. Supercond. 23, 4002405 (2013).

[5] D. Brown, A. Della Corte, C. F. Zignani, A. Gharib, D. Hagedorn, S. Turtu, and C. Rout, Cryogenic testing of high current by-pass diode stacks for the protection of the superconducting magnets in the LHC, AIP Conf. Proc. 711, 755 (2004).

[6] R. Denz and D. Hagedorn, Advances in Cryogenic Engineering Materials (Springer, Boston, 2000), Vol. 45, p. 375.

[7] R. Denz, A. Gharib, and D. Hagedorn, Radiation resistance and life time estimates at cryogenic temperatures of series 
produced by-pass diodes for the LHC magnet protection, AIP Conf. Proc. 711, 763 (2004).

[8] E. Floch et al., Irradiation of bypass diodes up to 2.2E14 Neutron/cm2 and $1.3 \mathrm{kGy}$ for the FAIR project, IEEE Trans. Appl. Supercond. 17, 2462 (2007).

[9] G. D'Angelo et al., Information for Authors, IEEE Trans. Appl. Supercond. 30, 4 (2020).

[10] E. Ravaioli, CLIQ, Ph.D. thesis, Univ. Twente, Enschede, 2015, http://doc.utwente.nl/96069/.

[11] High-Luminosity Large Hadron Collider (HL-LHC): Technical Design Report V. 1.0 (CERN, Geneva, Switzerland, to be published).

[12] T. T. Bhlen, F. Cerutti, M. P. W. Chin, A. Fasso, A. Ferrari, P. G. Ortega, A. Mairani, P. R. Sala, G. Smirnov, and V. Vlachoudis, The FLUKA code: Developments and challenges for high energy and medical applications, Nucl. Data Sheets 120, 211 (2014).

[13] A. Ferrari, P. R. Sala, A. Fasso, and J. Ranft, FLUKA: A multi-particle transport code, CERN, Geneva Report No. CERN-2005-10, INFN/TC_05/11, Report No. SLAC-R-773, 2005.

[14] R. G. Alia et al., Table of contents, IEEE Trans. Nucl. Sci. 65, 448 (2018).

[15] G. Lerner, R. G. Alia, M. S. Gilarte, A. Tsinganis, and F. Cerutti, Update on HL-LHC radiation levels on equipment in the IP1-IP5 LSS, presentation at the 9th $H L-L H C$ Collaboration Meeting, Fermilab, USA, 15 October 2019, https://indico.cern.ch/event/806637/contributions/ $3574371 /$.

[16] A. Thornton, CHARM facility test area radiation field description, Report CERN-ACC-NOTE-2016-004, 2016.
[17] A. Infantino, FLUKA Monte Carlo modelling of the CHARM facility's test area: Update of the radiation field assessment report No. CERN-ACC-NOTE-2017-0059, 2017.

[18] CHARM website http://charm.web.cern.ch, Accessed Nov. 2019.

[19] A. Will et al., Characterisation of the radiation hardness of cryogenic bypass diodes for the HL-LHC inner triplet quadrupole circuit, in Proc. 9th Int. Particle Accelerator Conf. (IPAC'18), Vancouver, Canada, 2018 (JACoW Publishing, Geneva, Switzerland, 2019), pp. 2620-2623, https://doi.org/10.18429/JACoW-IPAC2019-THPTS067.

[20] F. Ravotti, M. Glaser, and M. Moll, CERN Report No. TSNote-2005-002, 2005, https://edms.cern.ch/document/ $590497 / 1$.

[21] D. Wollmann et al., Characterisation of the radiation hardness of cryogenic bypass diodes for the HL-LHC inner triplet quadrupole circuit, in Proc. 10th Int. Particle Accelerator Conf. (IPAC'19), Melbourne, Australia (JACoW Publishing, Geneva, Switzerland, 2019), pp. 4268-4271, https://doi.org/10.18429/JACoW-IPAC2019-THPTS067.

[22] W. Shockley, The theory of p-n junctions in semiconductors and p-n junction transistors, Bell Syst. Tech. J. 28, 435 (1949).

[23] K. W. Böer and U.W. Pohl, Semiconductor Physics (Springer, New York, 2018).

[24] A. Holmes-Siedle and L. Adams, Handbook of Radiation Effects (Oxford University Press, New York, 1994).

[25] C. Leroy and P.-G. Rancoita, Principles of Radiation Interaction in Matter and Detection, 4th ed. (World Scientific, Singapore, 2016). 